\title{
Penile Gangrene by Non Metallic Objects: A Rare Aetiology in Adults
}

\section{Ravimohan*}

Department of Urology, Post Graduate Institute of Medical Education and Research, Nehru hospital Level II, 26, PGIMER , Sector 12, Chandigarh, India

\begin{abstract}
Penile strangulation from metallic and non-metallic objects has been reported since 1755 . The largest series of such cases was reported by Dakin in 1948, with the presenting age between 15 to 56 years. Penile strangulating objects are usually rings, nuts, bottles, bushes, wedding rings etc. in an adult, while in children they tend to be rubber bands threads or hair coils. In adults these constricting penile bands, whether expandable or non-expandable, are placed deliberately by the person himself for masturbation or by the female counterpart to prolong erection. Although condom catheters are more comfortable and less painful than indwelling catheters, they are not completely without risk. Various complications have been described in the literature, including skin irritation, maceration, ulceration, allergic reactions, urinary tract infections, fistula formation, penile edema, localized ischemia, and gangrene. We describe two cases of penile glans strangulation leading to auto amputation caused by a rubber band and another case of distal penile gangrene due to strangulation by tight condom catheter.
\end{abstract}

Keywords: Gangrene; Penis; Cavernosum; Condom catheter

\section{Case 1}

A 43 year old male presented to our OPD with complaint of lose of distal part of penis. 20 days back patient applied a perfume to his genitals, and in to the meatus followed which he developed irritation and pain in the penis. To decrease that pain he applied 10 rubber band to his penis. He removed all rubber bands after some time forgot to remove the one on corona. He was having obstructive voiding symptoms but still he ignored and was consuming analgesics for pain. After ten days he found that his glans is flabby and not having any sensation over that. Then he found to have a rubber band almost transecting the corona. While removing that rubber band glans also came out. At the time of presentation patient was having fever, pus discharge from the distal stump and leucocytosis. He was treated with antibiotics and debridement Psychiatric evaluation was normal. During debridement was found to have multiple small abscesses inside the corpus cavernosum which was drained and partial amputation was done $[1,2]$ (Figures 1 and 2).

\section{Case 2}

A 63 year old diabetic and hypertensive male patient admitted in

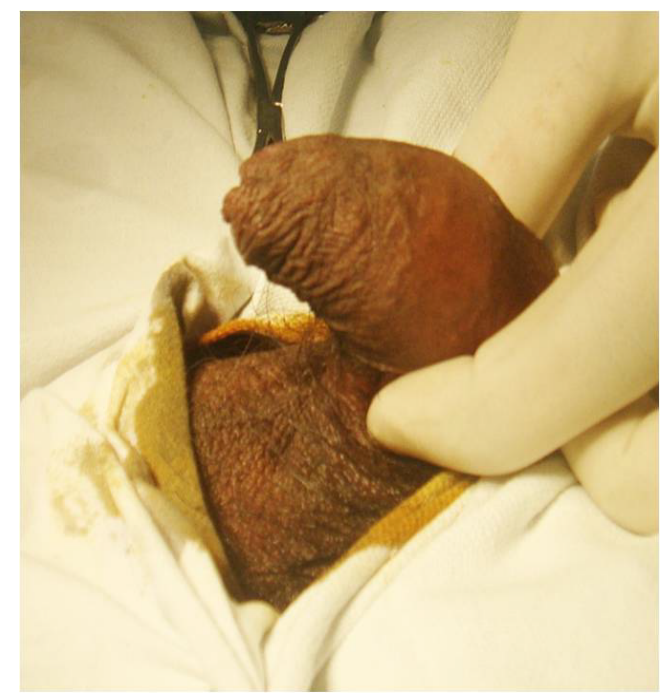

Figure 1: Redness over the penile shaft with covered prepuce.

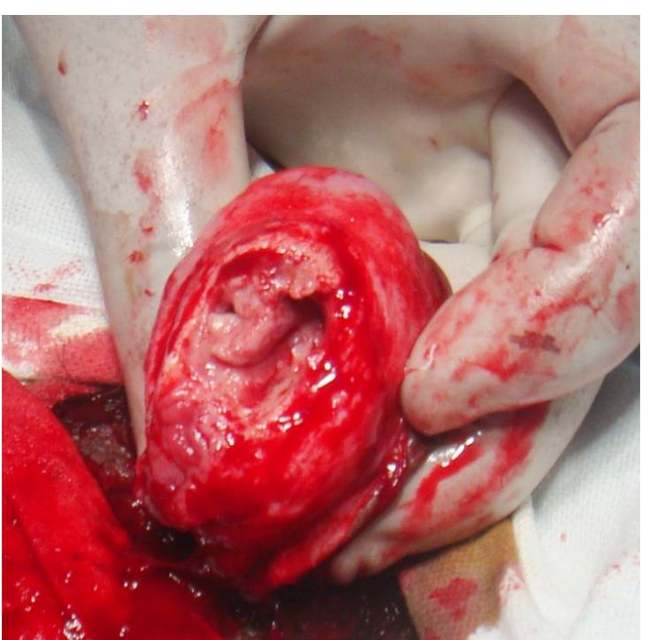

Figure 2: Following debridement raw area without any glans penis.

cardiology ward for angiography complained brown colored urine since one day. He was having right hemiperesis, and on condom catheter since 5 days. On removing the catheter found to have gangrene of the distal $2 / 3$ rd of the penis. Dorsal slit of prepuce was done for per urethral catheterisation. The gangrene got demarked in 3 days. Then partial amputation of the penis done and wound healed without any infection (Figure 3).

\section{Discussion}

Penile strangulation is a rare urological emergency. Gangrene of the penis is still rare. The progressive vaso-occlusive changes that result in penile gangrene are usually caused by diabetes mellitus,

*Corresponding author: Ravimohan, Department of Urology, Post Graduate Institute of Medical Education and Research, Nehru hospital Level II, 26, PGIMER, Sector 12, Chandigarh, India, E-mail: ravismi2003@yahoo.com

Received January 07, 2015; Accepted January 29, 2015; Published January 29 2015

Citation: Ravimohan (2015) Penile Gangrene by Non Metallic Objects: A Rare Aetiology in Adults. J Clin Case Rep 5: 484. doi:10.4172/2165-7920.1000484

Copyright: () 2015 Ravimohan. This is an open-access article distributed under the terms of the Creative Commons Attribution License, which permits unrestricted use, distribution, and reproduction in any medium, provided the original author and source are credited. 


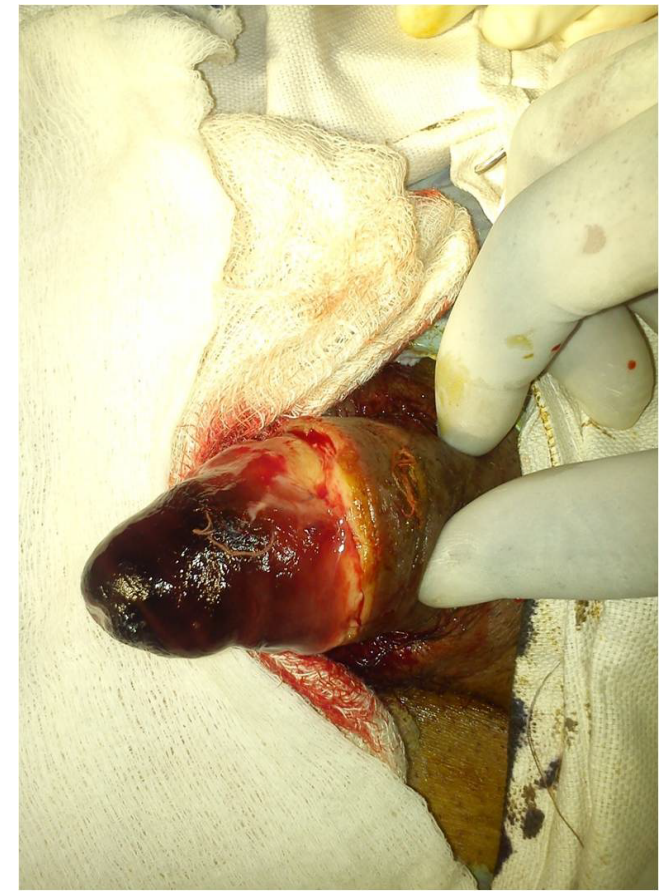

Figure 3: dry gangrene of the distal penis due to condom catheterization.

a buildup of calcium deposits owing to end-stage renal disease, or penile strangulation due to constricting devices [3,4] Agents of penile constriction, leading to strangulation and gangrene in some instances are varied but generally metallic objects, e.g. nuts, bolts, washers, hammer heads etc. are most commonly encountered. Consequently, several clinical syndromes can occur: from mild nonsignificant vascular obstruction that resolves after decompression to severe penile gangrene. Distal penile gangrene is seen very rarely in these scenarios. Few cases have been reported about partial penile gangrerne after applying condom catheter $[5,6]$

The diagnosis is obvious. A complete psychiatric evaluation is necessary if the history is suspicious as in the index patient one, and removal of the gangrenous phallus must be prompt to prevent septicaemia and/or tetanus. The consent for operation may be obtained from the close relatives. Psychiatric care is needed to rehabilitate the patient to accept the perineal urethrostomy in cases of total amputation. The neglected care as in the index case two, can also prove to be devastating. Delay in treatment is due to late presentation, the most severe consequence being complete penile gangrene. Bhat et al. [7] graded this injury into 5 categories, a useful guide to definitive treatment:

Grade I: Oedema of the distal penis. No evidence of skin ulceration or urethral injury.

Grade II: Injury to skin and constriction of corpus spongiosum, but no evidence of urethral injury. Distal penile oedema and decreased penile sensation.

Grade III: Injury to skin and urethra but no urethral fistula. Loss of distal penile sensations.
Grade IV: Complete division of corpus spongiosum leading to urethral fistula and constriction of corpora carvernosa with loss of distal penile sensations.

Grade V: Gangrene, necrosis, or complete amputation of distal penis. Grade V injury requires partial amputation as was done in both our cases. Delaying intervention usually requires more extensive surgery and increased the risk of wound complications. Thus, an aggressive surgical treatment initially is advocated. Simply observing these patients usually leads to subsequent liquefaction, infection, and urinary obstruction, which might require intervention with percutaneous supra-pubic tube drainage and debridement [8].

The second case also highlights an important aspect, that use of condom catheter should be judicious as it can be counterproductive if not properly taken care.

\section{Conclusion}

Partial penile auto amputation is very rare. Even non metallic elastic rubber band can also cause strangulation, gangrene and auto amputation of penis. Early debridement and partial amputation is important to preserve the penile stump as well as to achieve good cosmetic and functional results. Proper application and routine care of condom catheters is important in preventing this complication.

\section{References}

1. Ivanovski O Stankov O, Kuzmanoski M, Saidi S, Banev S, et al. (2007) Penile strangulation: two case reports and review of the literature. J Sex Med 4: 17751780.

2. Jayachandran S, Mooppan UM, Kim H (1985) Complications from external (condom) urinary drainage devices. Urology 25: 31-34.

3. Bour J, Steinhardt G (1984) Penile necrosis in patients with diabetes mellitus and end stage renal disease. J Urol 132: 560-562.

4. Lowe FC, Brendler CB (1984) Penile gangrene: a complication of secondary hyperparathyroidism from chronic renal failure. J Urol 132: 1189-1191.

5. Steinhardt G, McRoberts JW (1980) Total distal penile necrosis caused by condom catheter. JAMA 244: 1238.

6. Labanaris AP Zugor V, Smiszek R, Nützel R, Kühn R (2008) Dry gangrene of the penis induced by a bullring for sexual stimulation purposes ScientificWorldJournal 8: 896-900.

7. Bhat AL, Kumar A, Mathur SC, Gangwal KC (1991) Penile strangulation. Br J Urol 68: 618-621.

8. Weiner DM, Lowe FC (1996) Surgical management of ischemic penile gangrene in diabetics with end stage atherosclerosis. J Urol 155: 926-929. 